\title{
The emergence of scientific understanding in current ecological research practice
}

Forthcoming in History and Philosophy of the Life Sciences

Luana Poliseli ${ }^{1,2}$

${ }^{1}$ History, Philosophy and Biology Teaching Lab (LEFHBio), Institute of Biology, Federal University of Bahia (UFBA).

${ }^{2}$ National Institute of Science and Technology in Inter- and Transdisciplinary Studies in Ecology an Evolution

(INCT-INTREE)

luapoliseliramos@gmail.com

\begin{abstract}
Scientific understanding as a subject of inquiry has become widely discussed in philosophy of science and is often addressed through case studies from history of science. Even though these historical reconstructions engage with details of scientific practice, they usually provide only limited information about the gradual formation of understanding in ongoing processes of model and theory construction. Based on a qualitative ethnographic study of an ecological research project, this article shifts attention from understanding in the context of historical case studies to evidence of current case studies. By taking de Regt's (2017) contextual theory of scientific understanding into the field, it confirms core tenets of the contextual theory (e.g. the crucial role of visualization and visualizability) suggesting a normative character with respect to scientific activities. However, the case study also shows the limitations of de Regt's latest version of this theory as an attempt to explain the development of understanding in current practice. This article provides a model representing the emergence of scientific understanding that exposes main features of scientific understanding such as its gradual formation, its relation to skills and imagination, and its capacity for knowledge selectivity. The ethnographic evidence presented here supports the claim that something unique can be learned by looking into ongoing research practices that can't be gained by studying historical case studies.
\end{abstract}

Keywords: philosophy of science in practice, model building, visualization, skills, imagination, knowledge selectivity.

\section{Introduction}

Explaining and understanding natural phenomena is the raison d'être of science (de Regt and Dieks 2005). In early philosophy of science, the notion of understanding was routinely attributed to the notion of explanation (i.e. knowledge) (see Kvanvig 2003; Grimm 2006; Pritchard; 2009, 2014). More recently, the notion of understanding has gained prominence in philosophy of science and has been taken into philosophy of science in practice through the contextual theory of scientific understanding (de Regt, Leonelli and Eigner 2009; de Regt 2017). This article draws attention to the fact that much work in the philosophy of science remains insufficiently connected to the details of scientific practice (Ankeny et al. 2011). Although there are recent works connecting scientific understanding with ongoing research practices (see Leonelli 2009); the majority of examples ${ }^{1}$ used

1 I would like to draw special attention to the fact that not all investigations concerning understanding are based on historical case studies, there are increasingly investigations such as those provided by Leonelli (2009) that provide an account of scientific understanding via modelling practices and skills in contemporary biological 
both in mainstream philosophy of science and philosophy of science in practice come from historical case studies that provide limited information on the gradual formation of scientific understanding in ongoing processes of model and theory construction. Being historical, they are reconstructions created according to examples from the history of science.

A shift in focus may help shed light on the epistemic value of understanding in scientific practices while improving epistemological discussions in philosophy of science regarding the nature of understanding and the scientific activity of model-building. With this goal in mind, this investigation seeks to undertake this discussion from a different angle; using a detailed ethnographic study, it examines a current ecological research practice through the lens of de Regt's (2017) latest version of the contextual theory of scientific understanding. Such an approach complements historically oriented case studies concerning debates around scientific understanding by providing insights into the formation of scientific understanding. Through modeling practices (with special emphasis on the role of visualization), it looks at how understanding emerges and is achieved in real-time research, and how understanding leads to knowledge of explanandum.

The paper is organized as follows: Section 2 introduces the research context and the methodological aspects adopted in this investigation. Section 3 presents how the contextual theory of scientific understanding structures, organizes and assesses scientific understanding. Important to highlight here that this investigation does not tackle previous versions of the contextual theory (such as those presented in de Regt, Leonelli and Eigner (2009) Scientific understanding: philosophical perspectives book), instead, this investigation assesses the applicability of the contextual theory of scientific understanding as presented in de Regt's (2017) latest book Understanding scientific understanding. Section 4 introduces a case study from ecology and analyzes how the contextual theory can be applied to this ongoing scientific practice. Finally, Section 5 introduces a model that enables discussion as to how scientific understanding emerged in this current ecological practice.

\section{Research context and methodology:}

Philosophers pursue historical endorsement for their theories about science through the use of case studies (Bolinska and Martin 2019; Mizrahi forthcoming). The most prominent examples in debates regarding scientific understanding are elaborated from historical cases and may be insufficient to assess scientific understanding in ongoing scientific practices. In recent years, the use of case studies has become widespread, with the more apparent practice-orientation of mainstream philosophy of science (Knuuttila and Loettgers 2016; Mizrahi forthcoming). In the aim of presenting a different approach, the contextual theory of scientific understanding is applied to a current scientific practice in a biological context. The resulting investigation provides evidence for philosophy of science debates through a qualitative, ethnographically oriented philosophical work. It does so by accompanying the activities of an ecologist (occasionally referred to as scientists or modeler) during his scientific research: the development of a mechanistic model of pollination in agricultural systems, at the agricultural pole of Mucugê-Ibicoara, located in the Chapada Diamantina National Park, Brazil.

The data gathered for this analysis (i.e. statements, diagrams, models, tables, graphs and reports) was obtained through $>45$ meetings (with open interviews and questionnaires), which took place over a four-year period. In the first year, I gathered together with the ecologist during monthly meetings to develop a heuristics

research. 
set that would guide explanation construction and model building. This heuristics set was built in accordance with ecological theories and the new mechanistic philosophy of science; the dynamics of this development is depicted in Figure 1. Over the next few years, I stepped outside our mutual collaboration in order to evaluate how the scientist applied the heuristics set to model the phenomenon as well as to assess his understanding (for details on heuristics set application and mechanistic model development see Poliseli et al. forthcoming).

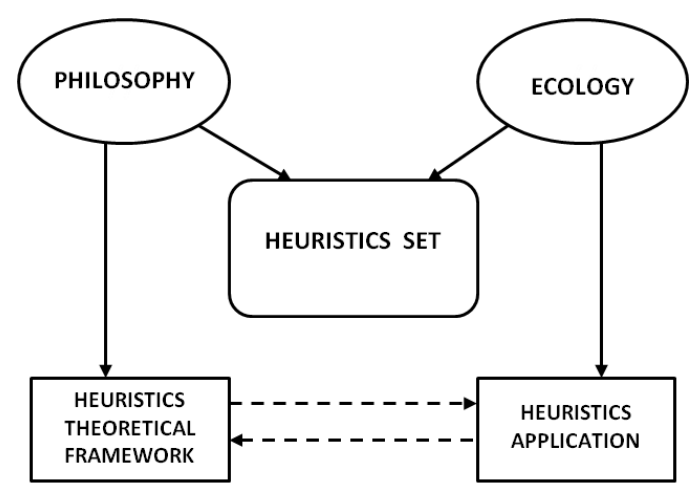

Figure 1: Collaboration dynamics between philosopher and ecologist to develop a heuristics set. The general conception of the heuristics set was constructed by combining the knowledge from theories in ecology and the new mechanistic philosophy of science. Its application was theoretically informed in the same way as its theoretical framework was empirically informed (see Poliseli 2018).

This case study sought to create a mechanistic model of this system and provide predictive scenarios. To do so, the heuristics set was developed in order to guide model-building, including, for example, a description of explanans and explanandum, an elaboration of mechanism sketches, identification of the systems conditions and variables, recognition of hierarchical structures, and so on (some main heuristics and its goals are exposed in Table 1). However, the goal of elaborating a mechanistic model was discarded during the research and replaced with a goal that was more suitable for such a complex phenomenon: the development of a conceptual framework that unifies mechanistic explanation, complex systems sciences and metacommunities theory (for details see Coutinho 2018). Ultimately, since it relates to the final product of the scientist's research project, the conceptual framework per se was not the main focus of this investigation. Instead, the main focus was upon the activities carried out prior to the conceptual framework, in other words, the elaboration itself, i.e. the formation of understanding in an ongoing research practice. The analysis of scientist practices by the light of the contextual theory of scientific understanding will be described in later sections.

Table 1: Heuristics set developed in interdisciplinary work engaging ecology and philosophy of science. The table presents main heuristics and their descriptions.

\begin{tabular}{|l|l|}
\hline Heuristics & Brief definition \\
\hline $\begin{array}{l}\text { Phenomenon } \\
\text { characterization }\end{array}$ & $\begin{array}{l}\text { The description of the phenomenon to be modeled and what will be } \\
\text { considered in its explanation. }\end{array}$ \\
\hline Mechanism sketch & $\begin{array}{l}\text { Diagrams that aim to establish the relationship between the theoretical } \\
\text { frameworks and the phenomena to be explained, whose purpose is to } \\
\text { construct a model that can explain the phenomenon. The two most }\end{array}$ \\
\hline
\end{tabular}




\begin{tabular}{|l|l|}
\hline & $\begin{array}{l}\text { important features of this sketch are its lack of information and its } \\
\text { disposable nature. Any mechanism sketch possesses gaps, gray and black } \\
\text { boxes, and may be discarded whenever necessary. }\end{array}$ \\
\hline $\begin{array}{l}\text { Hierarchical } \\
\text { structure }\end{array}$ & $\begin{array}{l}\text { A structure that seeks to identify and locate the [amount of] levels in } \\
\text { which the mechanism (or mechanisms) are organized and nested in the } \\
\text { phenomenon superstructure. This heuristics may enable visualizing the } \\
\text { interaction between different spatial and temporal scales. }\end{array}$ \\
\hline $\begin{array}{l}\text { Operational } \\
\text { components } \\
\text { distinction }\end{array}$ & $\begin{array}{l}\text { The goal is to distinguish the components and functions of the enabling } \\
\text { conditions within the mechanism and specify the relations and } \\
\text { boundaries between these components. If this information is not yet } \\
\text { present in the theoretical literature, it is highly recommended that one } \\
\text { carries out procedures of decomposition \& localization, forward \& } \\
\text { backward chaining, and synthetic \& analytic strategies to achieve this } \\
\text { goal. }\end{array}$ \\
\hline $\begin{array}{l}\text { Changing in } \\
\text { operational } \\
\text { components }\end{array}$ & $\begin{array}{l}\text { The attempt to exploit alternative scenarios and predict possible courses of the } \\
\text { system under investigation by modifying the operational components. }\end{array}$ \\
\hline \multicolumn{2}{|l|}{ Source: Poliseli (2018). }
\end{tabular}

\section{Scientific understanding}

Early philosophers of science used to analyze the notion of understanding with the notion of explanation. One way to do so was to assume understanding as an unproblematic counterpart of explanation, for instance, "one understands $A$ if and only if one can explain $A$ ". An independent analysis of understanding was deemed unnecessary because the theories of explanation were assumed to suffice for the discussion. Another way to do so was to assume understanding as a psychological dimension of explanation. As a psychological concept, understanding was not considered to be in the realms of philosophical elucidation, especially because philosophy was mainly interested in the rational and objective context of justification (Gijsbers 2013).

Philosophers of science's interest in the notion of understanding developed more recently, and the psychological dimensions of science, including notions such as the "feeling of understanding" and "grasp", are no longer viewed as being outside the boundaries of philosophy (Gijsbers 2013; Gopnik 1988). Consequently, philosophers started to doubt the veracity of the proposition "one understands $A$ iff one can explain A", which reflected discussions pertaining to the existence of understanding without explanation and, inversely, explanation without understanding (de Regt and Dieks 2005, Lipton 2009). This occurrence was the result of philosophers' diminishing aversion to recognizing science's psychological dimension while conceding that the differences between explanation and understanding could also rely on non-psychological features (Gijsbers 2013).

Even though scientific understanding has received increased attention, its role in scientific practices has only become recently addressed through accounts such as the contextual theory (de Regt and Dieks 2005; de Regt, Leonelli and Eigner 2009; de Regt 2017). In here, I will focus on the contextual theory of scientific understanding as presented in the book Understanding Scientific Understanding, where de Regt (2017) presents a general theory of scientific understanding that is pluralistic and independent of any specific model of explanation, allowing understanding to be achieved via different explanatory strategies. This theory asserts that to achieve scientific understanding, it is first necessary to understand the theories used to explain phenomena. 
Theories then must contain arguments that are intelligible for scientists to understand. In other words, "[o]nly intelligible theories allow scientists to construct models through which they can derive explanations of phenomena on the basis of the relevant theory” (de Regt 2017, p. 92). It is important to highlight that de Regt's intelligibility requirement relies on the following Criterion for Understanding Phenomena (CUP):

CUP: A phenomenon $P$ is understood scientifically if and only if there is an explanation of $P$ that is based on an intelligible theory $T$ and conforms to the basic epistemic values of empirical adequacy and internal consistency (ibid).

The contextual theory elaborates on the idea of variations in standards of intelligibility in scientific practice, asserting that scientific understanding should account for the contemporary and historical practice of science. Nonetheless, the intelligibility standards do not claim a status of exclusiveness and immutability because of the importance of changing contexts. Therefore, to achieve understanding is a macro-level aim (considering science as a whole), even though a scientist's view at the precise moment when understanding is achieved may be contextually situated at a meso- (e.g. scientific communities) or micro-level (e.g. individual scientists) (de Regt and Dieks 2005, p. 165).

Considering that one of science's universal epistemic aims is to understand phenomena and that scientific understanding requires intelligible theories, the contextual theory assumes intelligibility as a context-dependent feature concerning theoretical virtues as well as scientists' skills. Accordingly, scientists require intelligibility to be able to use theories in order to generate explanations and predictions. From such a perspective, these authors elaborate a Criterion for the Intelligibility of a Theory (CIT) that incorporates pragmatic and contextual features of understanding:

CIT: A scientific theory $T$ is intelligible for scientists if they can recognize qualitatively characteristic consequences of $T$ without performing exact calculations.

Therefore, in the contextual theory, a privileged status of particular standards of intelligibility (e.g. causality or unifying power) as necessary conditions for understanding is not assumed. Instead, it's argued that such intelligibility standards function as contingent tools to achieve scientific understanding in that they help scientists to intuitively discern the consequences of a scientific theory, thus fulfilling CIT requirements (de Regt and Dieks 2005). Although the contextual theory presents a normative scope for understanding across sciences, it has been primarily developed through examples from physics. Furthermore, the contextual theory is constructed using examples from the history of science. In other words, the scientific processes deployed in this theory are historical reconstructions created originally from the products of science - which may misguide the approximation of scientific understanding in ongoing practices. By adopting this theory, this paper is not only investigating if de Regt (2017) contextual theory can be applied to an ongoing scientific practice of a biological context, but is also inquiring if this theory is sufficient to explain the emergence of scientific understanding in real-time scientific practice. 


\section{Ecological research in practice: bees, mechanisms and frameworks}

Bees are the most important organisms for pollination in most regions of the globe (Klein et al. 2007). Worldwide, they are responsible for the pollination of approximately $70 \%$ of crops more than $80 \%$ of angiosperms. These groups of bees are usually involved in different ecological processes, such as water depuration, nutrient cycling, and the control of biological pests and pollination (Schleuning et al. 2015). All these ecological processes, namely ecosystem services, are intimately connected with agricultural food supplies for human societies and other biological communities.

In terms of their dynamics, agricultural systems are defined by an intricate set of ecological and nonecological characteristics. For instance, policies for landscape management that aim to suppress native vegetation may negatively affect several groups of pollinator species (Boscolo et al. 2017). Despite the importance, medium and long-term forecasts predict that intensive land use is not consistent with the stability of agricultural systems through time. In order to maintain ecosystem stability, land use will need to be restructured in a manner that is compatiblewith the conservation of biodiversity and ecosystem services. As a consequence, a connection exists between conservation and ecosystem services proposed via functional diversity of ecosystems services providers. The adoption of such a notion stems from distinct species' unequal contributions to the stability of ecological functions related to ecosystem services (Coutinho 2018). Taking this into account, the ecological phenomenon was defined by the scientist as the functional composition of autochthonous bee communities in agriculture systems, i.e. investigate how native bee communitiesorganize themselves in agricultural systems, concerning their functional roles such as pollination. 


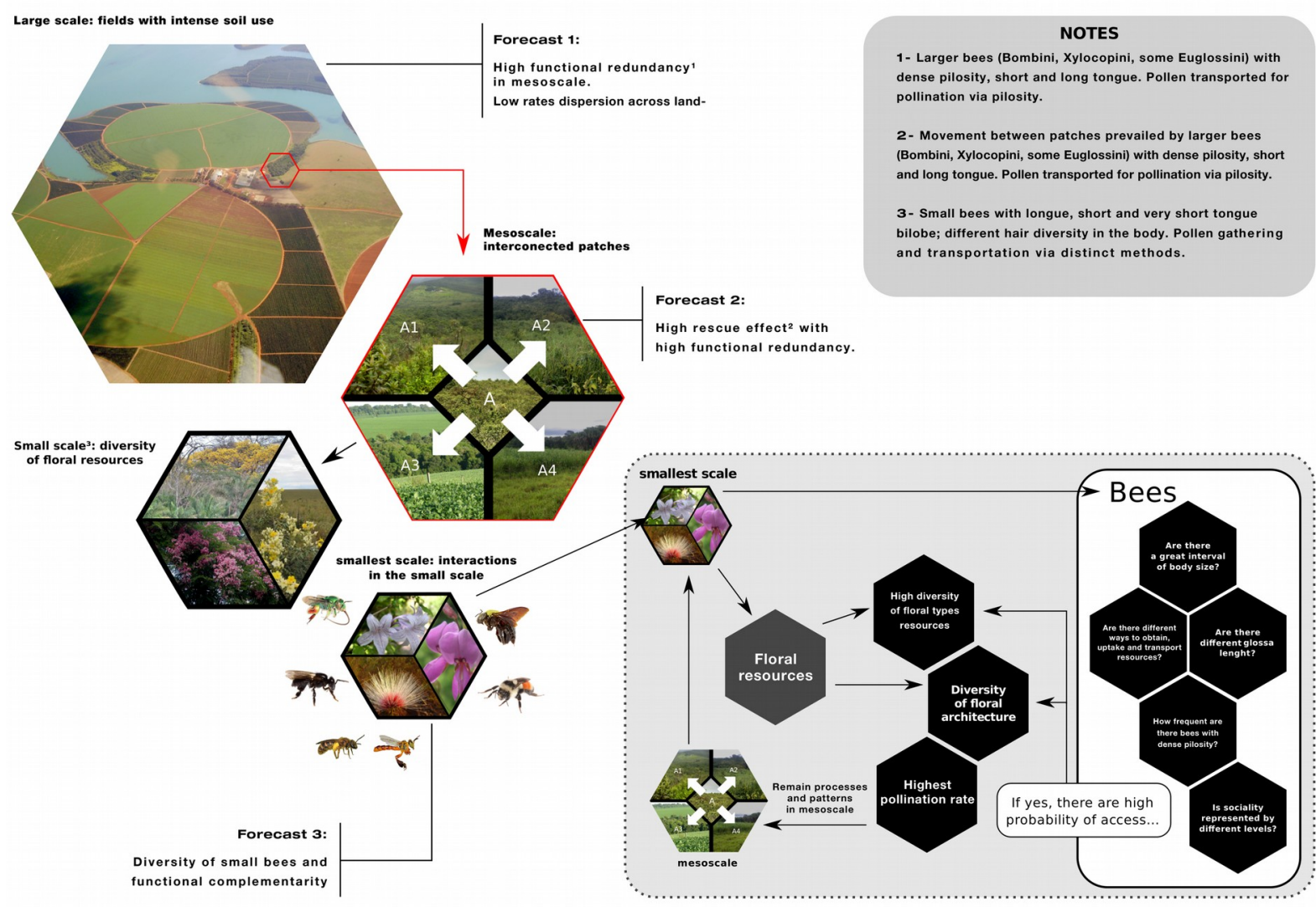

Figure 2: mechanism sketch developed by the scientist to represent the ecological phenomenon and derive forecasts for bee-plant interaction (explanation vide text) (designed by Diogo Lula Amorim). Source Coutinho (2018).

In an agricultural system such as the aformentioned example, the patch interconnection (i.e. vegetation fragmentation) is anelement that enables higher levels to function as a mechanism. A landscape may possess several heterogeneous patches, from native and secondary vegetation to agricultural fields. Each of these patches has various dynamics and impacts differently on the pollination system, which in turn grants uniqueness in terms of the bee-flower interaction's dynamics (Figure 2). In a top-down view, this mechanism recognizes a four-level hierarchy. Although neither the elements nor the activities are established, it is still possible to identify different forecasts (or expected behaviors) for levels related to the bees' features. For instance, at the smallest level (forecast 3 ) the diversity of small bees works as functional complement to the diversity of larger bees moving between the patch fragments that are interconnected at the mesoscale. At forecast 2, the distance of the fragment influences pollinators' movements: the shorter the distance between the fragments, the larger the dispersion of the pollinators amid the landscape. Meanwhile, in terms of habitat diversity, the more diverse the habitats, the larger the floral resources at the small scale. The management stability of a viable population of pollinators and the ecosystem service they provide, needs a synchronism between plant flowering and animal presence. For pollen gathering (dotted gray box) there must be synchronism between phenology and foraging; otherwise, there is no possibility of gathering. If the synchronism is positive, then the encounter between flower 
and pollinator will happen through behavioral displays and structural features (Figure 2, Notes 1-3) that enable recognition and interactions.

This case study in ecology addressed the most relevant ecological processes in an attempt to explain the functional diversity of bees in agricultural systems. To do so, these agricultural systems were re-evaluated from a complex systems perspective (see Solé and Gooldwin 2000; Boccara 2004; Mitchel 2009; Cadotte et al. 2011; Filotas et al. 2014), complemented by the theoretical-methodological framework of mechanistic explanation. To explain this phenomenon, a study of the literature highlighted the most relevant spatio-temporal scales. Since the forms of interaction between these variables are not always clear in ecology, it's important to arrive at a more satisfactory level of explanation, by reducing existing gaps, looking for intersections between theories and, considering spatial and environmental features along with attributes of the chosen phenomenon's life history (Scientist, personal communication). The analysis of the scientist's practices by the light of the contextual theory of scientific understanding will be described bellow.

\subsection{Contextual theory and conceptual tools in ecological practice}

The core idea of de Regt's contextual theory revolves around intelligibility²: "Intelligibility [is] the value that scientists attribute to the cluster of qualities of a theory (in one or more of its representations) that facilitate the use of the theory” (de Regt 2017, p. 40). Thus, a theory may be intelligible in different ways and this is only possible because theories are historically content- and context-dependent. Therefore, some tools allow a theory to be more intelligible than others, consequently, facilitating scientists' understanding. Included among these tools are visualization, causal reasoning, visualizability, unificationist notions, and mathematical index. Their adequacy with respect to the scientific practice of this case study will be depicted below.

Using visualization as a tool for understanding is a common practice in the history of science. According to Machamer et al. (2000) and de Regt (2017), seeing is the most important way for humans to perceive the world, which can be attributed to our ontogenetic and phylogenetic development. It is thus understandable that scientists are inclined to use visualization as a tool for scientific understanding, once our sensory experience provides bases for intelligibility which are then extended to realms beyond sense perception (de Regt 2017, pp. 257-258). It is important to highlight that visualization is a possible tool for achieving understanding, however it is not a necessary condition. As such, in a (not so) hypothetical situation, a scientist with limited visual capability is also able to gain understanding.

According to Bechtel and Abrahamsen (2005), diagrams of complex mechanisms are preferred to theoretical representations because they can inform the spatial organization of mechanisms, making them easier to track. The contextual theory (de Regt 2017, p. 258) adds that this does not mean that diagrammatic reasoning can be applied easily and immediately. As perceived in the following statement of the scientist ${ }^{3}$, this is not an easy task. At the same time that each attempt to develop a mechanism sketch helped the scientist with solutions, it also helped to perceive gaps regarding the scientific literature underlying the system. This outcome reveals the

2The contextual theory of understanding makes no difference between theories, hypothesis, and principles. The starting point is that all of them are statements, and these statements can be reliable according to its intelligibility (de Regt 2017). This is in agreement with Giere (2004) when he says that there is no reason for such analysis because the terms 'theory', 'laws' and 'principles are used broadly in scientific practice and in metalevel discussions about sciences. 3 The statements exposed are authorized. 
challenges in building diagrammatic representations of complex systems and, based on this ethnographic evidence, underscores the persistent attempts required to develop mechanisms sketches that allowed to gradually grasp the phenomenon.

In the first proposition of the "mechanism sketch" I realized that I could not arrive at a minimally reasonable scheme of communication of my phenomenon and of the relevant variables for such. [...]. With each new sketch, new challenges, and solutions, two dimensions of the process of construction of scientific knowledge that led me to lead a process of full immersion in the scientific literature to address the gaps that were gradually perceived. [SCIENTIST, personal communication]

In the case study, the epistemic value visualization for model building was widely perceived throughout the scientist's activity. One activity that deserves attention was the development of mechanism sketches, examples of its wide application are presented in Figure 3.

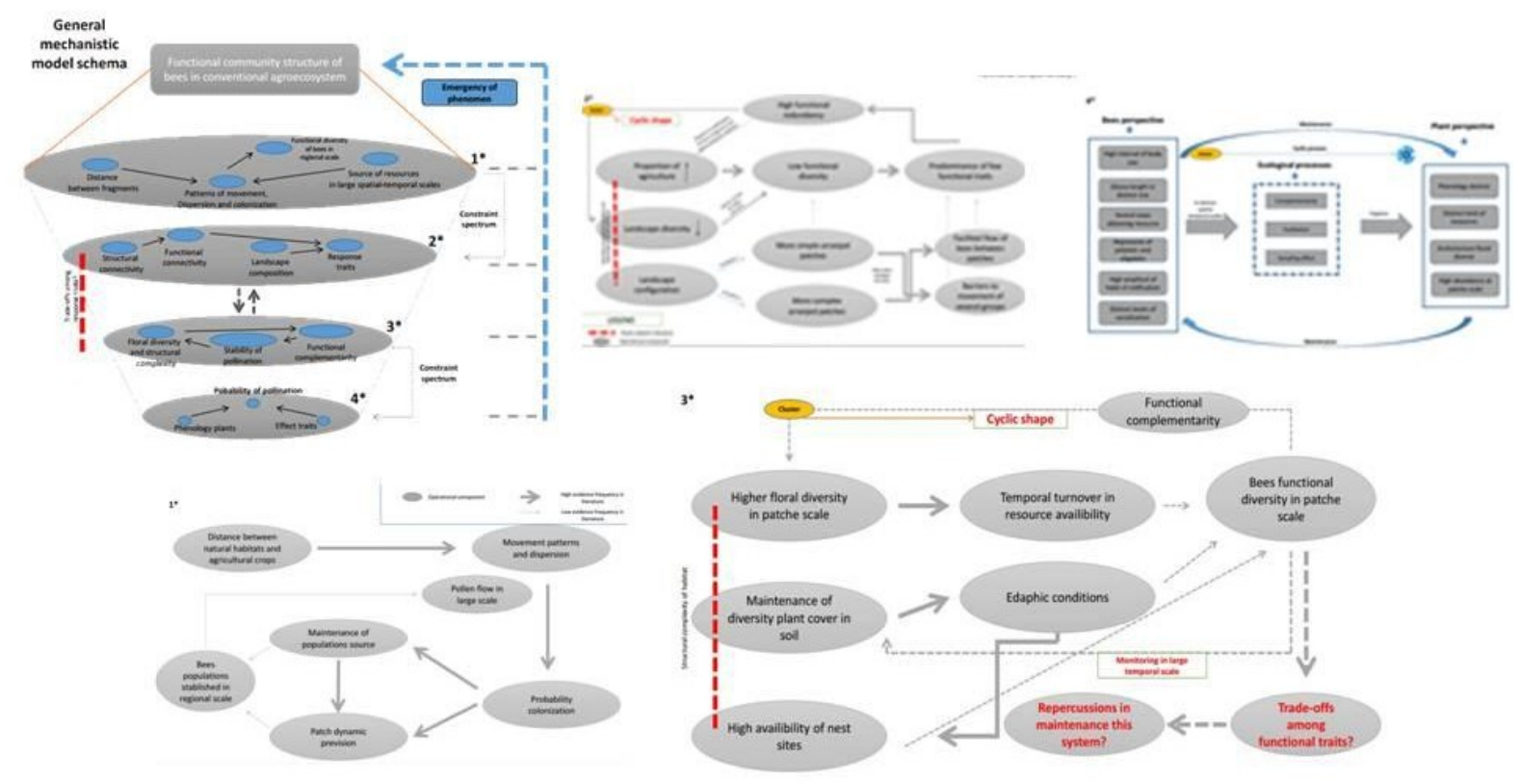

Figure 3: Five examples from the $>21$ sketches, diagrams and mechanisms developed by the ecologist during his research. Hierarchical model elaborated with 4-level structure and subsequent mechanisms of each level. $1^{*}$ mechanisms of the regional scale. $2^{*}$ mechanism of the landscape scale. $3^{*}$ mechanism of the patch scale. $4^{*}$ mechanism of the flower scale. All of them heuristics-based and further discarded. Source Poliseli (2018).

Recognizing the amount of diagrams created by the scientist, one cannot deny the important role they had for the model's development and the construction of an explanation, seeing that biologists intervene on models in order to reach explanations of the phenomenon in question (Leonelli 2009). In this sense, this agrees and confirms that models work as objectual tools to improve theories (Morgan and Morrison 1999; Knuuttila and Merts 2009). In this specific case study, visualizability had a fundamental role for scientific understanding through model construction, even though this role was provisional when compared to the final framework put 
forward by the modeler. The power of this conceptual tool is further confirmed by the modeler with respect to the precise shape of the mechanism:

In wider theoretical models, the general format of the mechanism, when dealing with a more complex structure, [also] contributes. [...] The definition of the typology is crucial in understanding the magnitude that certain processes may have in generating patterns of bee functional diversity. This heuristics pointed to a core of hypotheses that need an empirical evaluation with great potential to generate advances in the understanding of the relation between functional diversity aspects and ecosystem properties [SCIENTIST, personal communication].

Since the construction of a mechanistic model was the main goal of the model building, the causal reasoning tool was, unsurprisingly, strongly recognized in the scientist's practice. Beyond the causal mechanistic approach previously mentioned, an example of causal reasoning took place when the scientist developed a schema to determine which system variables could be considered as information in the modeling (Figure 4).

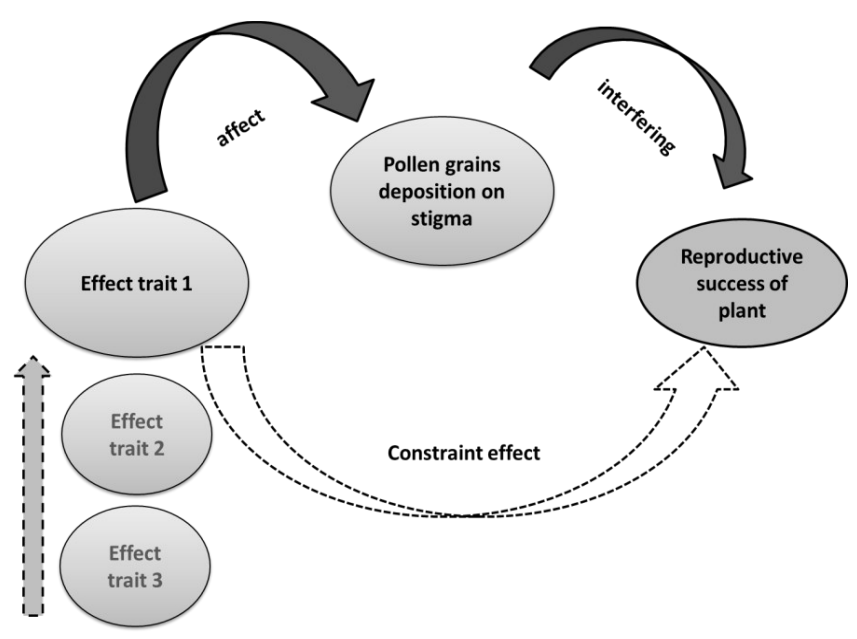

Figure 4: Diagram indicating steps for the recognition of relevant effect traits related to the pollination in a system. An effect trait $(1,2,3)$ will be relevant if, and only if, its exclusion implicates changes in the deposition of grains of pollen on the stigma. This will consequently reduce the amount of fruits and/or seeds of the target plant species, which will deviate from the maximum potential of the species. This constraining effect of the effect trait on the reproductive success of the plant should guide the selection of relevant traits in the analysis. Source: Coutinho (2018).

According to the modeler statement, "In the first proposition of the mechanism sketch I realized that I could not arrive at a minimally reasonable scheme of communication of my phenomenon, and of the relevant variables for such". "Scheme of communication" can be read precisely as a means of developing a complete mechanism that explicitly identifies the elements and causal relations of the system. Such a reading enables us 
to make inferences about what Machamer et al. (2000) call productive continuity. The continuity for Machamer et al. concerns the causal relations between the elements of a mechanism that enable it to produce an event or byproduct (i.e. the phenomenon) and can be easily tracked as a conceptual tool. In our case study, the modeler was not able to identify such productive continuity in the first sketches due to several gaps that existed in the ecological literature. The completion of such black and gray boxes was only possible due to his knowledge of mainstream ecological literature. During this occurrence, the understanding of his phenomenon was becoming more apparent, as expressed in the scientist's more detailed schema. Ultimately, causal reasoning led him to understanding because it revealed what he took to be the underlying structure of the world:

[...] I cannot construct a conceptual model if I do not know the set of variables and conditions of interaction between these variables that are relevant in the proposition of this model. This does not mean that we should be able to begin the proposition of the model by knowing all the relevant variables - this would take away one of the great virtues of the art of modeling: a gradual refining of our theoretical constructs about the functioning of the world. This heuristics [enabling conditions] is the backbone of this work since it allows a constant search in the literature for ecological variables (drivers) of paramount importance in explaining the phenomenon and its zones of intersection and influence. [...] This heuristics "opened my mind" to a more critical view in the ecological literature, searching for variables of paramount importance for the proposition of the model (SCIENTIST, personal communication)

Grasping the productive continuity of the mechanisms allowed the scientist to explore alternative scenarios and predict possible paths of the system under investigation (Figure 5). This was achieved by modifying some of the enabling conditions. Such research practice is acknowledged by Leonelli (2009, p. 192) that "explanation in biology is always obtained through direct intervention on models of the phenomenon to be explained". In harmony with, Figure 4 exposes two alternative scenarios that the modeler developed while considering elements of the metacommunity theories as enabling conditions, capable of providing the necessary conditions for the mechanism's productive continuity. Left, combination of species sorting and patch dynamics. In this layout the patches are well connected and present high structural complexity of the vegetation. In this case, the agricultural activity has been recently introduced into the system, there is no vegetation suppression. Hence, there is a high supply of resources for pollination. Right, combination of species sorting with mass effect. There is a predominance of agriculture at the scale of the landscape and the remnant patches will serve as strongholds for small bees. The extinction of landscape will act as a filter for the dispersion of bees. At the local scale, the structural characteristics that will select non-soil, oligolectic and small bees due to their response traits will be maintained. The larger bees will be responsible for a large part of the pollen flow between patches, increasing the chance of service stability at the meso- and macroscale. Some groups of crop plants will still continue to be favorred by the presence of large bees. 


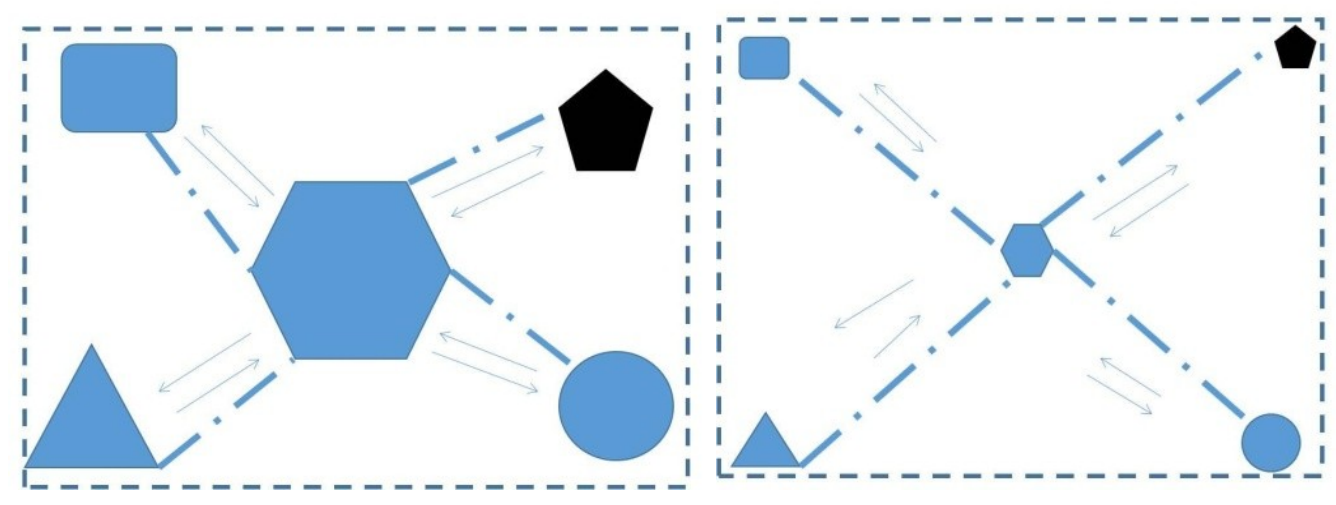

Figure 5: Two of $>7$ schemas developed to predict distinct ecological scenarios for the phenomenon. In each figure, the scientist combined different scenarios according to metacommunity theories. Polygons represent distinct patches with different dynamics; arrows represent interactions between patches and dotted lines represent bees' movements.

This activity allowed for the alteration of some system attributes (such as diversity traits), beyond those usually proposed by empirical literature, allowing for the interaction with wider ecological frameworks and reflection upon a diversity of scenarios. This is in accordance with the contextual theory which states that causal reasoning enhances the scientist's ability to predict how systems will behave under particular conditions. In Woodward's (2003) account, scientific understanding can also be achieved by answering questions about the behavior of the system being investigated. Even though there are different standards for intelligibility, the contextual theory suggests the following criteria:

CIT: A scientific theory $T$ (in one or more of its representations) is intelligible for scientists (in context $C$ ) if they can recognize qualitatively characteristic consequences of $T$ without performing exact calculations.

Scientific explanation in the ecological realm must possess a predictive nature (explanatory and anticipatory) if it is to be of use in management. Predictions must be based on a theoretical framework (explicit or implicit) that is decisive for its aims and limitations (on the absence of a theory, no prediction is possible). Consequently, the understanding of processes responsible for the phenomena of interest not only helps in the construction of explicative models, but also contributes to their predictive accuracy (Mouquet et al. 2015). This assertion is aligned with one of the epistemic goals of science, namely prediction. In the contextual theory of scientific understanding, the ability to predict, i.e. to recognize qualitative consequences of a theory, means that the theories are intelligible to scientists. Thus, in this ecological case study, the understanding assessment also fits the CIT. One of the main virtues of variations in terms of standards of intelligibility is that one can accommodate the manifold ways in which understanding is achieved in scientific practice. According to this notion, if scientists understand a theory, the theory is intelligible to them;which was exactly perceived in this case study.

The unificationist conceptual tool brings an interesting aspect to a discussion about the biological field. Biological sciences are extremely disunified, presenting a diversity of epistemic cultures (Leonelli 2009). The 
knowledge produced possesses various forms: mechanistic, causal reasoning, historical narrative, descriptive, functional, mathematical, representational, and categorical (ibid. 2009). Since the nature of the data and the methods of analysis are so diverse, it is not surprising that scientists try to solve this puzzle in a piecemeal manner which is also what happened in the case study. In choosing which variable was the most significant to model, the scientist created a table that included an incredible variety in the nature of data, all of which was collected using different instruments and strategies, and therefore was analyzed accordingly (see Poliseli 2018). The idea of this table was to gather information to recognize how they function in the pollination service in this agricultural system. In addition, an effort was made to combine different theoretical propositions, as stated:

[...] It is no novelty that ecological phenomena are hierarchical and that there are several levels of interaction between scales in the hierarchy, which may be spatial, temporal or spatiotemporal. This heuristics [hierarchical structure] was applied when I understood the potential that two metacommunity models could help me in proposing our conceptual model. I realized here that both could make great contributions, provided I used the most appropriate scale in using the forecasts of each of these models. THEY COULD BE COMPLEMENTARY IN THE EXPLANATION OF THE PHENOMENON. It was here that I realized that the discourse of plurality in scientific explanation was fully consistent with my phenomenon: it was all a matter of scale [...] [SCIENTIST, personal communication, emphasis added by the modeler].

It is undeniable that unification played a major role in the development of explanation and model construction. Nonetheless, its epistemic role was not only limited to the empirical approach regarding the diversity of data nature. It also embraced the theoretical scope employed by the scientist. For instance, the conceptual framework developed was mainly scaffolded according to three distinct theories from different domains, i.e., mechanistic explanation, complex systems science and metacommunity theories.

It is also possible to associate these activities with the types of understanding suggested by Gijsbers (2013). According to Gijsbers, there are at least two types of understanding. The first type of understanding would be achieved according to the scientist's acknowledgment of the phenomenon's causal relations (explanation-understanding or vertical connections). The second type would be achieved based on the scientist's knowledge of the phenomena's unification aspects (unification-understanding or horizontal connections). There is enough evidence in this case study to assert that these two types of understanding occurred, however, it is not possible to agree they took place in this specific order, viewed that the scientist's activities were occurring concomitantly. The only certainty about this practice is that the knowledge concerning the phenomenon gradually increased according to its complexity and the need to elaborate more complex explanations. However, this does not mean that his understanding through unification only occurred later on. This is acknowledged by Khalifa and Gadomski (2013) when they assert that the causaland the unificationist models are appropriate for different contexts and therefore require a plurality of explanatory relations. These activities were so intricately related to each other that even the possibility of a distinct separation was merely viable for the theoretical purposes of trying to bring order to a somewhat chaotic endeavor. 
This section revealed that de Regt's (2017) contextual theory provides us with normative tools to assess scientific understanding. This theory is indeed sensitive to the context, as it can be applied both to historical case studies in physics as well as to current ecological research practice. However, it seems that this theory is still bound to explanatory strategies (see Knuuttila and Merz 2009) regardless of efforts to add a psychological aspect to it. Qualitative scenarios and intuitive judgment are not enough to explain the emergence of understanding, which also relies upon psychological and cognitive aspects. This lack indicates the need for future investigations. For instance, how does understanding emerge in distinct domains of knowledge? What exactly are qualitative predictive scenarios and how are they developed? How does intuitive judgment behave? The following section will contribute to these debates by answering how does understanding emerge?

\section{The emergence of scientific understanding in ongoing ecological research practice}

This section argues that the ecological case study suggests a remarkable account of the formation of scientific understanding in current research processes. Recall that the methodological innovation of this article is to address the formation of scientific understanding in an ongoing research project through in-depth ethnographic research. Figure 6 provides a unique account of how scientific understanding emerges in real-time scientific practice by providing empirical evidence for debates that are long-time theoretical. The general idea of this model is that scientific understanding was achieved through two distinct stages: first, it relied upon the scientist's basic skills, in here considered as background knowledge and abilities; and secondly, through the use of imagination. One interesting aspect exposed by this model is that both forms of understanding provided a reliable source of knowledge for subsequent scientific and ecological activities, suggesting a role of knowledge selectivity. Some core tenets of this process, such as stages of understanding, imagination, and selectivity feature will be addressed in following subsections.

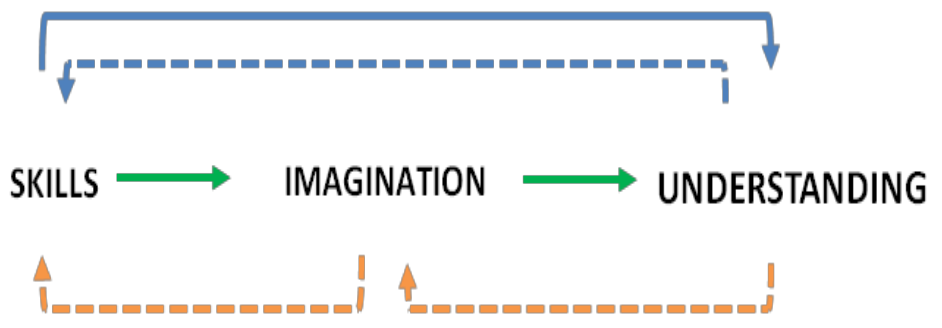

Figure 6: Model representing the emergence of scientific understanding in current ecological research practice. Note the different ways that lead to understanding, in other words, degrees of understanding are reached through the gradual development of skills and imagination. Full arrows represent direct relations between the boxes. Dotted arrows represent feedback relations between the boxes. Colored arrows suggest distinct stages of the process of emergence of scientific understanding (explanation vide text).

5.1. Emergence of understanding and its relation to stages of understanding 
The idea that understanding occurs in degrees is widely acknowledged (Kvanvig 2003). Nonetheless it often remains unclear how these degrees develop and how they should be assessed. The most common idea asserts that the more detailed the knowledge, the deeper the understanding. Take for example this sentence: “degrees of understanding simply correspond to different amounts of knowledge. An agent understands better why $p$ in so far as she knows more about why p" (Sliwa 2015, p. 71). This section will reveal, among other things, that degrees of understanding do not reduce to the amount of knowledge as defined by Sliwa (2015). Instead, understanding is intimately related to an agent's ability to interact with the information received and the complexity of this information (Section 5.3). To avoid confusion, in this context, degrees of understanding refer to gradual stages of understanding, and are intrinsically related to the complexity of the information. I will developed this idea.

Before I explain the Figure 6 model, it is important to consider that biologists ought to exercise specific epistemic skills in order to materially intervene on and reason about the world (Leonelli 2009, p. 190). Epistemic skills can be defined as

[...] the abilities to carry out a number of activities in order to increase one's understanding of reality. They may be partly innate, such as the skill of drawing an object (which depends to some degree on the talent of the individual attempting such action), yet they are most often acquired through the imitation of others and/or through experience, for instance by trial or error. [...] the notion of skill concerns also the means and manners by which action is undertaken (ibid 2009, p. 2000).

In the case of scientific research, epistemic skills can be distinguished in theoretical skills, performative skills, and social skills (ibid). Theoretical skills relate to the capacity to manipulate various expressions of theoretical knowledge (i.e. abstract models, theories, concepts). Performative skills are acquired only through practice, through the direct interaction with the environment, and enables the scientist to exploit material resources such as laboratory equipment, specimens, and so on. And, social skills is the ability of researchers to conform to existing social standards (e.g. the ability to engage with research and express their works according to a given research community) (for more details see Leonelli 2009). Considering the Figure 6 model, one of the propositions defended here is that the understanding achieved in the ecological case study emerged from a gradual process. At all stages of understanding, the scientist $(S)$ is considered as an epistemic agent who possesses highly technical skills coupled with a theoretical background that enables him to make reliable decisions about his object of study. Thus, epistemic skills here relates to theoretical skills and performative skills only. Once it is assumed that knowledge can be understood in terms of successful action plus warrant (Williamson 2000; Hawley 2003), these become important features.

At the first stage of understanding (Figure 6, full blue arrow), the scientist's epistemic skills were sufficient for him to achieve understanding of the phenomenon (upper full blue arrow). This became possible once it is assumed that non-explanatory information can form the basis for understanding (e.g. Lipton 2009; Gijsbers 2013; Kelp 2015; de Regt and Gijsbergs 2016). Therefore, these abilities worked as mediators, which allowed the scientist to gain understanding by means of expertise and technical effort. In science, it might be the case that the understanding achieved at this stage was also a final product, but in this model it is represented as a 
first stage of understanding. For instance, if the ecologist's final goal was to explain his phenomenon by means of a simple diagram, he would have reached understanding with his first sketch (Figure 2). However this was not the case. While developing mechanisms sketches, the modeler perceived theoretical gaps in the literature, which required completion (see Section 4.1). Connecting with this Section's model, this allowed the scientist to proceed to a second stage of understanding.

The understanding that emerged in the first stage scaffolds knowledge and expertise that will be further used in analyses and experiments, resulting in an epistemic improvement of the scientist's skills (Figure 6, dotted blue arrow). As a result, the scientist is prepared for a next stage of understanding. Based on the case study, this can be exemplified by the several definitions of explanans and explanandum developed by the scientist during his research. Initially, explanandum was defined simply as the "diversity patterns of insect pollinator in agricultural systems". A second characterization sought to narrow down the phenomenon by selecting a category of pollinator and a specific type of diversity: "functional composition of autochthonous bee communities in agricultural systems". A third definition was related to a specific agricultural system and ecosystem service: "the functional structure of an autochthonous bee community, as well as the maintenance of its pollination services in an agricultural system”. For each new definition, new elements were gathered or excluded in order to help the scientist understand the system being modeled. What can be learned from this case is that, the gradual improvement of the delimitation of the system being investigated reflected its amount of complexity. This model thus illustrates how scientific understanding is transient. Once again, theoretical knowledge and technical expertise (combined into skills) allowed the scientist to understand his phenomenon.

It is worthy to note that these different stages dos not necessarily relate to different types of understanding. For instance, in biological practices Leonelli (2009) suggests three types of understanding: theoretical understanding, embodied understanding, and integrated understanding. Theoretical understanding concerns the understanding of biological phenomena that is achieved "through recourse to theoretical commitments and skills, with performative skills and commitment playing a subsidiary role” (ibid, p. 204). Embodied understanding uses theoretical skills and commitment to develop performative skills with the goal to interact and intervene in the phenomenon (theoretical commitment having a subsidiary role). And, integrated understanding is a balanced interaction between theoretical and embodied knowledge. While it would feel prompt to associate theoretical knowledge to the first stage, embodied understanding to the second stage, and the overall process to an integrative understanding; this is not necessarily assumed in this case study. One of the reasons why Figure 6 model does not account for distinct types of understanding is because it might be possible that theoretical, embodied and integrative understanding occur at any stage of the process. Once the scientific practice is not assumed as a linear process, such stages will not necessarily follow a sequence, instead, it will occur according to a given research goal (see Section 5.3).

\subsection{Emergence of understanding and its relation to imagination}

There are several strategies through which scientists can recognize qualitative characteristics consequences of $T$ without performing exact calculations (de Regt 2017). Considering that $S$ is not always in a position to causally interact with the phenomenon (Ylikoski 2009), abilities (developed in previous stage of the model) qualify $S$ to consider the phenomenon in a way in which laboratory instruments aren't required for 
analysis. These strategies are products of mental activities, named "imagination" or "imaginative processes" and are key features to the emergence of understanding (Figure 6, green arrows). This imagination will act basically through intuitive judgment and thought experiments, producing counterfactual scenarios that will provide 'qualitative consequences of $T$ '. I will elaborate.

To connect the contextual theory with the aspect of imagination, recall that:

[...] skill and intuitive judgment play a central role in the process of achieving scientific understanding. If a theory is intelligible to scientists because its theoretical qualities match their skills, they can reason "intuitively" with it. Like our everyday intuitive skills, scientists' skills are the outcome of a complex learning process in which their evolved cognitive capacities interact with the environment in which they find themselves (that is, the historical and disciplinary context of their science) (de Regt 2017, p. 110).

According to de Regt (2009, 2017), skills and judgment cannot be reduced to rule-following procedures because they change according to the historical, social or disciplinary context. Such skills will depend on a scientist's given theory and its pragmatic virtues. For instance, a model's construction relies upon a specific theoretical framework that demands the modeler possess specific skills concerning its theoretical properties. Such skills may vary from expertise techniques (e.g. lab work, data collection) to grasping and intuitive judgment.

De Regt (2017) elaborates upon Gigerenzer's (2007) psychological notion of intuitive judgment, which instead of viewing intuitive judgments as obscure, sees them as being produced by heuristics that are usually developed during an evolutionary process of adaptation to the environment. Gigerenzer (ibid) acknowledges the reliability of intuitions and their role in decision-making processes. He defines intuition as judgment that arises immediately in consciousness without full awareness of underlying causes (for contrary views see Kahneman 2011).

The intuitive judgment referred to here acts through mental action that enables the modeler to assess counterexamples. This mental action only occurs due to the capability of imaginative processes. Imagination or imaginative processes are fundamental higher mental actions that may also occur through thought experiments, metaphors and utopias (Tateo 2016, 2020). One type of thought experiment is anticipatory understanding. In anticipatory understanding, when causal interaction with an object is not possible, relevant inferences may be made about future consequences of some event or series of events regarding the explanandum (Ylikoski 2009). Counterfactual situations offer predictions of the phenomenon without causal intervention. They do so by creating 'internal mental models' (Waskan 2006) that suffer the judgment of intuition, which is consonant with the criterion for the intelligibility of a theory at the contextual theory of scientific understanding. With respect to the ecological case study, the counterfactual scenarios elaborated by $S$ were reflected through the heuristics 'change in the operational distinction'. In this heuristics, $S$ developed internal mental models capable of creating counterfactual scenarios that then became the external models represented in Figure 5. It is worthwhile to state that success under certain counterfactual circumstances is a necessary condition for knowledge (Hawley 2003). 
In this sense, anticipatory understanding is considered here as a type of thought experiment, and more specifically, as a counterfactual thought experiment. As with any thought experiment, it deliberately and purposively appeals to imagination (de Mey 2006). In order to avoid a slippery notion, it has be used with caution (Hawthorne 1991), manipulating one factor at time (de Mey 2006). In this manner, anticipatory understanding can be a powerful tool. For instance, reasoning processes of thought experiments should involve the construal of weighed explanations that happen in two steps: contrast and counterfactuals. The idea of weight is used to determine whether one cause of the phenomenon is more important than another. Thus, the contrast specifies a situation in which the explanandum is compared to mental scenarios, which then drastically reduces the number of possible causes for the following counterfactual reasoning (de Mey 2006). Statements such as those below contribute to the idea that counterfactual thought experiments were indeed used by the scientist as scaffolding to achieve understanding of the phenomenon:

The heuristics [elaboration of mechanism sketch] has awakened me to this theoretical gap that existed in me in relation to the phenomenon that I have studied. With each new sketch, new challenges, and solutions, two dimensions of the process of construction of scientific knowledge that led me to lead a process of full immersion in the scientific literature to address the gaps that were gradually perceived. Each new reading enables me to review my sketches (often not materialized, but purely mental), and I will reaffirm my understanding of my phenomenon and the potential contributions that this research can provide [SCIENTIST, personal communication]

Considering the ability of humans to mentally account for real and imagined situations as well as the predictive power of thought, internal mental models can be structural, behavioral, or functional analogues to real-world phenomena (Craik 1943). In this sense, mental models are constructed to be reasoned with (Thagard 2010). They can therefore be useful in providing explanations (Johnson-Laird 1980, 1983), as explicitly noted in the modeler's statement above. This is clearly apparent in the case study where the scientist combined species sorting with mass effect principles to develop predictive scenarios, as discussed in the previous section. The explicit intent was to suggest adjustments to some elements of the phenomenon in order to predict possible modifications in the system under investigation.

Another example of mental accounts was the modification of the heuristics role during the scientific practice. Table 1 reveals the epistemic role of some main heuristics as empirical activities. These heuristics were developed at the beginning of the ecologist's research undertaken with the philosopher. When considering Table 2 together with the content created by the modeler at the end of this research practice, the modification of the heuristics from empirical activities to mental activities is explicitly apparent. Instead of actions, they have become questions to be answered.

Table 2: Contribution of each heuristics in the construction of the ecologist's theoretical framework (according with the ecologist).

\begin{tabular}{|l|l} 
Heuristics & Brief definition \\
\hline
\end{tabular}




\begin{tabular}{|c|c|}
\hline $\begin{array}{l}\text { Phenomenon } \\
\text { characterization }\end{array}$ & $\begin{array}{l}\text { What do we want to explain/predict? What are the boundaries of our } \\
\text { question of interest? }\end{array}$ \\
\hline Mechanism sketch & How do we communicate our framework in a clear and intelligible way? \\
\hline $\begin{array}{l}\text { Hierarchical } \\
\text { structure }\end{array}$ & $\begin{array}{l}\text { How many, and which, hierarchical levels are most relevant to } \\
\text { understanding the phenomenon of interest? }\end{array}$ \\
\hline $\begin{array}{l}\text { Operational } \\
\text { components } \\
\text { distinction }\end{array}$ & $\begin{array}{l}\text { Which, if any, response variables and predictors be synthesized into more } \\
\text { ecologically relevant components? }\end{array}$ \\
\hline
\end{tabular}

The development of external models, represented in Figure 5, clearly shows internal mental models undergoing modification to become external models. While complementing the idea that successful scientific cognition combines internal representations with external representations, there is an important distinction: internal mental models are not understanding (Ylikoski 2009). Rather, as mediators, they enable the formation of understanding, which subsequently allows one to reach a second level of understanding (Figure 6, green arrows).

\subsection{Emergence of understanding and its relation to knowledge selectivity}

Once understanding was achieved, it transformed itself once more into knowledge that served as a basis for theoretical expertise, skills and imagination (Figure 6, orange arrows), supporting the idea of an ephemeral and transient character of understanding. Such a dynamic allows the modeler to improve his skills and to further develop increasingly complex counterexamples. However, it is important to note that the gradually improved ability to understand complex phenomenon does not mean that scientific understanding is akin to cumulative process, an ad infinitum addition of information from one level to another. On the contrary, scientific understanding is also a selective and refinement process of information gathered. It's important to recall that intelligibility enables scientists to use theories in order to generate explanations and predictions. Being intelligible is a context-dependent feature related to the scientist's skill and theoretical virtues (de Regt and Dieks 2005). In this sense, scientific understanding is value-laden because it can be grounded in the idea that human developmental processes, such as learning, creating, planning, imagining, building, problem-solving, inventing, etc., can lead to both generative and destructive outcomes (see Tateo 2016). As seen in the case study, the scientist was capable of choosing between diagrams, theories, and models that best fit his conceptual framework.

Looking at Figure 6 model, some questions arise: how many levels of understanding are possible? If scientific understanding occurs in degrees, is there a higher or greater understanding that can be achieved? Do the different standards of intelligibility suggest different standards of scientific understanding? The existence of degrees of understanding is consistent with the idea of different standards of intelligibility. However, the existence of degrees of understanding, and therefore, degrees of intelligibility, does not mean that degrees will occur in every scientific practice. Considering that scientific practice concerns explanation or model construction, and that the achievement of understanding is content- and context-related, the number of stages 
might be connected to the explanandum. The more complex a phenomenon being studied, the more degrees of understanding and intelligibility may occur.

An important difference between understanding and scientific understanding, which might appear tautological, but it is useful to recall, is that the latter is tacitly immersed in a scientific context where the practices of a scientific community operate: "understanding can only be qualified as "scientific" when obtained through the skillful and consistent use of tools, instruments, methods, theories, and/or models” (Leonelli 2009, p. 190). Thus, any level of understanding will be intrinsically related to a given research goal. As such, there is no greater or higher understanding, only the understanding that is supposed to be achieved according to $S$ 's investigation. Undoubtedly, there will be some kind of increase in complexity, once the understanding in one stage is embodied, becoming expertise and skills for future stages. This is aligned with Leonelli (2009, p. 199) that "in the case of biological understanding, the quality of the understanding displayed by a researcher will depend on his or her acquisition of appropriate background knowledge as well as expertise in handling instruments, models, and theories that make it possible to produce and apply any scientific explanation". However, this does not preclude the possibility that the so-called second stage takes place before the so-called first stage. The same takes place with kinds of understanding, as discussed in Section 5.1. The model should, therefore, not be seen as an oversimplified linear event but also as proposing an account of a waterfall or spiral phenomenon product of a creative thinking process (see Boehm 1988; Ebert 1994; Gupta and Bhatia 2012).

This section discussed how the emergence of scientific understanding takes place in ongoing research practice. By representing this emergence through a model, main features pertaining to the formation of understanding was explored, i.e. the use of skills and imaginative processes. The ethnographic evidence presented provided sufficient information to sustain that scientific understanding can be gradually achieved through different stages. In this case study, two stages were recognized. Important aspects of this process are its knowledge selectivity and refinement feature. After each stage, understanding assigns reliable knowledge for subsequent scientific and ecological activities. This also suggests that in order to achieve scientific understanding, one needs to have at least a minimum amount of scientific knowledge, in other words, the information thought to contribute to understanding must be accessible to the epistemic agent (de Regt 2017).

Despite the contextual theory's normative character and its applicability to current case studies, it seems that de Regt's (2017) latest version of the contextual theory cannot sufficiently explain how scientific understanding actually emerges. Such a conclusion supports the claim of this article that historical case studies may only tell one part of the story. The other part may very well be written through the use of ethnographic tools to access and assess real-life case studies in the attempt to reach domains beyond those tied to ready-made explanations.

\section{Conclusion}

The aim of this article has been to develop an account of the gradual emergence of scientific understanding in an ongoing ecological research practice. While de Regt (2017) contextual theory of scientific understanding is based on careful case studies of scientific practice, much of the debate has been shaped by historical examples that provide only limited information with respect to cognitive processes and other details related to constructing 
and revising models in practice. The methodological innovation of this study has been to combine the contextual theory with ethnographic research of an ongoing research process and to address the formation of understanding through an analysis of evidence gathered through empirical case study (see also Leonelli 2009).

The outcomes of this investigation are twofold. First, the case study validates core assumptions of the contextual theory. It confirmed the crucial role of visualization in scientific understanding by tracing how the construction and revision of visual sketches guided the development of models and theories. Secondly, the case study's results enabled the development of a model tracing the emergence of scientific understanding in current research practices. These outcomes complement discussions surrounding the nature of understanding, as seen through a fine-grained analysis of the process of scientific understanding, which is a result of the formation of epistemic skills and imaginations that are iteratively transformed in current research practice of model and theory construction. While acknowledgeding the absecense of several information pertaining to each box of the model (i.e. skills, imagination), future empirical investigation is required. The model's overall simplicity does not do justice to the complexity of its achievement. Nevertheless, this simplification is a starting point for a reconstruction of scientific understanding through empirical evidence of case studies. Further research is still necessary to test whether the Figure 6 model can guide philosophical analysis regarding the emergence of understanding in other cases of current scientific practices, and how the emergence of understanding relates to types of understanding.

To conclude, a core feature of this study is its qualitative ethnographic method, based on long-term collaboration with a researcher in ecology. This method has important strengths in facilitating an in-depth analysis of the formation of understanding in a complex real-life case and providing a nuanced picture of the function of tools such as the visual sketches discussed in Section 4. At the same time, reliance on one particular case study necessarily makes the generalization of Section 5 hypothetical. Even though this model's very specific context makes it difficult to claim generality (see Levin 1992), historians and philosophers of science that propose general hypotheses of how science works, usually rely on case studies (Bishop and Trout 2002). To quote Mizrahi (forthcoming, p. 10), "Since philosophy of science is about science, by definition, it means that philosophers of science tend to argue for meta-scientific conclusions [that] are general in nature”, thus, case studies of ongoing scientific research might as well be used as evidence for meta-scientific conclusions that are supposed to apply to science as a whole.

\section{REFERENCES}

Ankeny, R., Chang, H., Boumans, M. and Boon, M. (2011). Introduction: philosophy of science in practice. European journal for philosophy of science 3(1): 303-7.

Baumberger, C. (2014). Types of understanding: their nature and their relation to knowledge. Conceptus 40.

Bechtel, W. and Abrahamsen, A. (2005). Explanation: a mechanist alternative. Studies in History and Philosophy of Biological and Biomedical Sciences 36(2):421-41.

Bishop, M. A. and Trout, J. D. (2002). Reason in the balance: an inquiry approach to critical thinking. Second edition. Indianopolis: Hackett Publish Company.

Boccara, N. (2004). Modeling complex systems. New York, NY: Springer.

Boehm, B. W. (1988). A spiral model of software development and enhancement. Computer 21(5): 61-72. 
Bolinsca, A. and Martin, J. D. (2019). Negotiating history: contingency, canonicity, and case studies. Studies in History and Philosophy of Science Part A. https://doi.org/10.1016/j.shpsa.2019.05.003

Boscolo, D., Tokumoto, P. M., Ferreira, P. A., Ribeiro, J. W. and Santos, J. S. (2017). Positive responses of flower visiting bees to landscape heterogeneity depends on functional connectivity levels. Perspect. Ecol. Conserv. 15: 18-24.

Cadotte, M. W., Carscadden, K. and Mirotchnick, N. (2011). Beyond species: functional diversity and the maintenance of ecological processes and services. Journal of Applied. Ecology. 48: 1079-1087.

Cane, J. and Schiffhauer, D. (2003). Dose-response relationships between pollination and fruiting refine pollinator comparisons for cranberry (Vaccinium Macrocarpon [Ericaceae]). American journal of botany 90: $1425-32$.

Corbet, S. A. (1998). Fruit and seed production in relation to pollination and resources in bluebell, Hyacinthoides Non-Scripta. Oecologia 114(3): 349-60.

Coutinho, J. G. E. (2018). Diversidade funcional de abelhas em sistemas agrícolas: aportes teóricos, empíricos e epistêmicos. Originally presented as doctorate dissertation, Universidade Federal da Bahia. Salvador, Ba. Craik, K. J. W. (1967). The nature of explanation. CUP Archive.

De Mey, T. (2006). Imagination’s grip on science. Metaphilosophy 37(2): 222-39.

de Regt, H. W. (2009). Understanding and scientific explanation. In H. W. de Regt, S. Leonelli, and K. Eigner (Eds.). Scientific Understanding: philosophical perspectives (pp. 21-42). Pittsburgh: University of Pittsburgh Press.

de Regt, H. W. (2017). Understanding scientific understanding. New York: Oxford University Press.

de Regt, H. W. and Dieks, D. (2005). A contextual approach to scientific understanding. Synthese 144(1): 137170 .

de Regt, H. W. and Gijbergs, V. (2017). How false theories can yield genuine understanding. In S. Grimm, C. Baumberger and S. Ammon. (Eds.). Explaining understanding: new perspectives from epistemology and philosophy of science (pp. 50-57). Pittsburgh: University of Pittsburgh Press.

de Regt, H. W., Leonelli, S. and Eigner, K. (Eds.). Scientific Understanding: philosophical perspectives. Pittsburgh: University of Pittsburgh Press.

Ebert, E. S. (1994). The cognitive spiral: creative thinking and cognitive processing. Journal of Creative Behavior, 28(4): 275-290.

Fantl, J. (2008). Knowing-how and knowing-that. Philosophy Compass 3(3): 451-470.

Filotas, E., Parrott, L., Burton, P. J., Chazdon, R. L., Coates, K. D., Coll, L., Haeussler, S., Martin K., Nocentini, S., Puettmann, K. J., Putz, F. R., Simard, S. W. and Messier, C. (2014). Viewing forests through the lens of complexity systems science. Ecosphere 5(1): 1-23.

Giere, R. N. (2004). How models are used to represent reality. Philosophy of Science, 71(5): 742-752.

Gigerenzer, G. (2007). Gut feelings: the intelligence of the unconscious. London: Penguin Books.

Gijsbers, V. (2013). Understanding, explanation, and unification. Studies in History and Philosophy of Science Part A 44(3): 516-22.

Gopnik, A. (1988). Explanation as orgasm. Minds and Machines, 8: 101-118.

Grimm, S. R. (2006). Is understanding a species of knowledge? The British Journal for the Philosophy of Science 57(3): 515-35. 
Gupta, S. and Bhatia, P. K. (2012). Cognitive spiral model: a framework approach. International Journal of Advances in Engineering, Science and Technology (IJAEST), 1, 2, 194-199.

Hawley, K. (2003). Success and knowledge-how. American Philosophical Quarterly 40(1): 19-31.

Hawthorn, G. (1991). Plausible worlds: possibility and understanding in history and the social sciences. Cambridge University Press.

Johnson-Laird, P. N. (1980). Mental models in cognitive science. Cognitive Science 4(1): 71-115.

Johson-Laird, P. N. (1983). Mental models. Cambridge: Harvard University Press.

Kahneman, D. (2011). Thinking fast and slow. London: Allen Lane.

Kelp, C. (2015). Understanding phenomena. Synthese, 192(12): 3799-3816

Khalifa, K., and Gadomski, M. (2013). Understanding as explanatory knowledge: The Case of Bjorken Scaling. Studies in History and Philosophy of Science Part A 44(3): 384-92.

Klein, A-M., Vaissière, B. E., Cane, J. H., Steffan-Dewenter, I., Cunningham, S. A., Kremen, C. and Tscharntke, T. (2007). Importance of pollinators in changing landscapes for world crops. Proceedings of the Royal Society, 274:303-313.

Knuuttila, T. and Merts, M. (2009). Understanding by modeling: an objectual approach. In H. W. De Regt, S. Leonelli, and K. Eigner (Eds.). Scientific Understanding: philosophical perspectives (pp.146-168). Pittsburgh: University of Pittsburgh Press.

Knuuttila, T. and Loettgers, A. (2016). Contrasting cases: the Lotka-Volterra model times three. Pp. 151-178 in T. Sauer and R. Scholl (Eds.). The Philosophy of Historical Case Studies. Basel: Springer.

Kvanvig, J. (2003). The value of knowledge and the pursuit of understanding. New York: Oxford university press.

Leonelli, S. (2009). Understanding in biology: the impure nature of biological knowledge. In H. W. De Regt, S. Leonelli, and K. Eigner (Eds.). Scientific Understanding: philosophical perspectives (pp.189-209). Pittsburgh: University of Pittsburgh Press.

Levin, S. A. (1992). The Problem of pattern and scale in ecology: the Robert H. MacArthur award lecture. Ecology, 73, 1943-1967.

Lipton, P. (2009). Understanding without explanation. In H. W. De Regt, S. Leonelli, and K. Eigner (Eds.). Scientific Understanding: philosophical perspectives (pp.43-63). Pittsburgh: University of Pittsburgh Press.

Machamer, P., Darden, L. and Craver, F. C. (2000). Thinking about mechanisms. Philosophy of science, 67, 125.

Mitchell, M. (2009). Complexity: a guided tour. New York, NY: Oxford University Press.

Mizrahi, M. (forthcoming). The case study method in philosophy of science: an empirical study. Perspectives on Science. Available on PhilArchive: https://philarchive.org/archive/MIZTCS

Morgan, M. S. and Morrison, M. (1999). Models as mediators: perspectives, on natural and social science. Cambridge: Cambridge University Press.

Mouquet, L., Lagadeus, Y., Devictor, V., Doyen, L., Duputié, A., Eveillard, D., Faure, F., Garnier, E., Gimenez, O., Huneman, P., Jabot, F., Jarne, P., Joly, D., Julliard, R., Kéfi, S., Kergoat, G., Lavorel, S., Le Gall, L., Meslin, L., Morand, S., Morin, X., Morlon, H., Pinay, G., Pradel, R., Schurr, M., Thuiller, W. and Loreau, M. (2015). Predictive ecology in a changing world. Journal of Applied Ecology, 52:1293-1310.

Poliseli, L. (2018). When ecology and philosophy meet: constructing explanations and assessing understanding in scientific practice. Originally presented as doctorate dissertation, Universidade Federal da Bahia. Salvador, Ba. 
Poliseli, L. (2020). Book review [Resenha]: de Regt, H. Understanding Scientific Understanding, OUP, 2017. Principia: an International of Epistemology, 24(1): 239-245.

Poliseli, L. and El-Hani, C. N. (2020). Imagination in science. In: L. Tateo (ed.) A theory of imagining, knowing and understanding, (pp.65-84). Switzerland: Springer Briefs.

Pritchard, D. (2009). Knowledge, understanding and epistemic value. Royal Institute of Philosophy Supplement 64: 19-43.

Pritchard, D. (2014). Knowledge and understanding. In A. Fairweather (Ed.) Virtue epistemology naturalized. Synthese Library. Studies in Epistemology, Logic, Methodology, and Philosophy of Science, 366. Springer. 2014. Ryle, G. (1949). The concept of mind. London; New York: Hutchinson's University Library.

Schleuning, M., Fründ, J. and García, D. (2015). Predicting ecosystems functions from biodiversity and mutualistic networks: an extension of trait-based concepts to plant-animal interactions. Ecography (Cop.), 38: 380-392.

Sliwa, P. (2015). Understanding and knowing. Proceedings of the Aristotelian Society, 115: 57-74.

Solé, R. V. and Goodwin, B. (2000). Signs of life: how complexity pervades biology. New York, NY: Basic Books.

Tateo, L. (2016). What imagination can teach us about higher mental functions. Psychology as the Science of Human Being: The Yokohama Manifesto: 149-64.

Tateo, L. (2020). A theory of imagining, knowing and understanding. Switzerland: Springer Briefs.

Thagard, P. (2010). How brains make mental models. In L. Magnani, W. Carnieli, \& C. Pizzi (Eds.) Modelbased reasoning in science and technology: abduction, logic and computational discovery (pp. 447-461). Berlin: Springer.

Waskan, J. A. (2006). Models and cognition: prediction and explanation in everyday life and in science. Cambridge: The MIT Press.

Williamson, T. (2002). Knowledge and its limits. Oxford University Press.

Ylikoski, P. K. (2009). The illusion of depth of understanding in science. Scientific Understanding: Philosophical Perspectives: 100-119. 